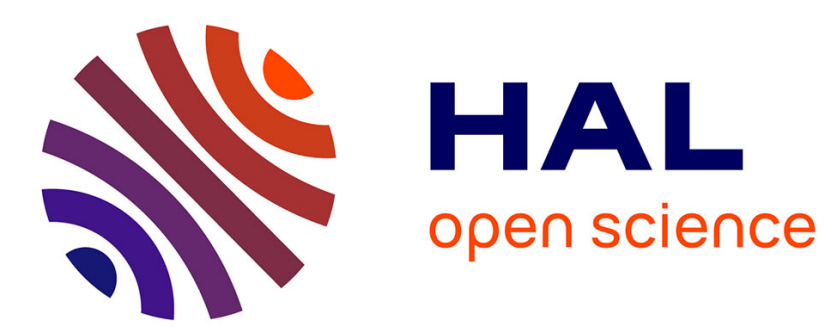

\title{
Utilisation par l'informatique et traitement statistique d'un fichier ampélographique
}

Jean-Michel Boursiquot, Marie-Paule Faber, Olivier Blachier, Paul Truel

\section{To cite this version:}

Jean-Michel Boursiquot, Marie-Paule Faber, Olivier Blachier, Paul Truel. Utilisation par l'informatique et traitement statistique d'un fichier ampélographique. Agronomie, 1987, 7 (1), pp.1320. hal-00884964

\section{HAL Id: hal-00884964 https://hal.science/hal-00884964}

Submitted on 1 Jan 1987

HAL is a multi-disciplinary open access archive for the deposit and dissemination of scientific research documents, whether they are published or not. The documents may come from teaching and research institutions in France or abroad, or from public or private research centers.
L'archive ouverte pluridisciplinaire HAL, est destinée au dépôt et à la diffusion de documents scientifiques de niveau recherche, publiés ou non, émanant des établissements d'enseignement et de recherche français ou étrangers, des laboratoires publics ou privés. 


\title{
Utilisation par l'informatique et traitement sta- tistique d'un fichier ampélographique
}

\author{
Jean-Michel BOURSIQUOT, Marie-Paule FABER $\left({ }^{*}\right)$, Olivier BLACHIER $\left({ }^{*}\right)$ \& Paul TRUEL $\left({ }^{* *}\right)$ \\ E.N.S.A., Chaire de Viticulture, F 34060 Montpellier Cedex \\ (*) E.N.S.A., Chaire de Biométrie, F 34060 Montpellier Cedex \\ (**) I.N.R.A., Domaine de Vassal, F 34340 Marseillan-Plage
}

RÉSUMÉ

\begin{abstract}
L'ampélographie reste un problème d'actualité chez Vitis vinifera L. En effet, reconnaître et identifier une variété est souvent une opération délicate. Ceci est dû au grand nombre de cépages existants et à l'importance de la fluctuation présente au sein de chacun d'eux.

Une aide à la reconnaissance est proposée en utilisant par des moyens informatiques un fichier ampélographique portant sur 5226 clones (2076 variétés). Ce fichier a été réalisé au Domaine de VAsSAL (I.N.R.A., 34340 Marseillan-Plage). Les descriptions intéressent 75 caractères, ce qui représente 272 modalités. Après sauvegarde de ces données sur Mini 6, un programme en Pascal a été mis au point permettant de questionner ce fichier et d'obtenir ainsi des listes d'hypothèses pouvant correspondre aux échantillons testés.

Une analyse statistique (A.F.C.) de ces données a permis de mettre en évidence les caractères ampélographiques les plus importants et d'obtenir une image de la population de Vitis vinifera $\mathrm{L}$. contenue dans ce fichier. Deux directions dispersent ainsi le mieux les variétés :

- la première oppose les cépages de cuve (petites baies) présentant généralement une densité des poils couchés forte, aux variétés de table (grosses baies), souvent glabres ;

- la seconde met en opposition les variétés à caractères juvéniles marqués (pigmentation anthocyanique prononcée, densité des poils dressés importante) avec des cépages plus évolués.
\end{abstract}

Mots clés additionnels : Ampélographie, Vitis vinifera L., programme de tri, analyse des données, caractères juvéniles.

\section{Computerization and statistical analysis of ampelographic data.}

Ampelography remains a problem of current concern. The great number of varieties of Vitis vinifera $\mathrm{L}$. and the great variability within cultivars cause difficulty in identifying and recognizing varieties. A method for aiding recognition of varieties using computerized ampelographic data has accordingly been developed. Data were collected from the Research Station of VASSAL (I.N.R.A., 34340 Marseillan-Plage). 5226 clones representing 2076 cultivars were described using 75 characters (with 272 values). The descriptions were recorded in a Mini 6 computer and a programme was developed to examine the file and obtain hypothetical listings which might agree with an unknown sample. Statistical analysis (C.F.A.) of these data showed which ampelographic characters were most useful and gave a picture of the population of Vitis vinifera $\mathrm{L}$. The population of varieties was dispersed in two main directions. The first opposed wine-production cultivars with small berries and often a high density of prostrate hairs to table varieties (large berries), which are usually hairless. The second opposed varieties with marked juvenile characters (high anthocyanin colour, high density of erect hairs) to more developed varieties.

Additional key words : Ampelography, Vitis vinifera L., sorting programme, data analysis, juvenile characters.

\section{INTRODUCTION}

La confrontation avec un échantillon de vigne pose pour un ampélographe le problème de la reconnaissance et de l'identification. Il se trouve parfois dans l'impossibilité d'identifier un échantillon.

Plusieurs causes peuvent alors expliquer cet échec :
- l'échantillon n'est pas suffisamment représentatif,

- l'ampélographe ne se souvient pas de la variété représentée par l'échantillon (limite de la mémoire),

- l'échantillon représente réellement un cépage inconnu ou nouveau pour l'ampélographe (limite de la connaissance). 
Dans les 3 cas l'ampélographe n'arrive pas à formuler d'hypothèses ou à émettre la bonne hypothèse. En effet, le processus de reconnaissance passe nécessairement par la formulation d'une hypothèse qui doit être suivie de sa vérification. Cette dernière étape peut être réelle ou intellectuelle suivant que l'on dispose ou non d'éléments de références : collections, herbiers (BRANAS, 1959).

Si l'hypothèse d'identité émise entre un échantillon et une variété est inexacte l'ampélographe doit s'efforcer de ne pas l'accepter lors de la vérification. En effet, rassembler sous une même dénomination 2 cépages différents peut avoir des conséquences économiques importantes en favorisant par exemple certains abus.

Aussi pour aider les personnes confrontées à ces problèmes on a imaginé un système destiné à pallier le manque d'hypothèses et à réduire si possible le nombre des mauvaises.

Ce système repose sur l'établissement d'un fichier de référence dans lequel les cépages sont décrits suivant un certain nombre de caractères. On compare alors les caractéristiques de l'échantillon inconnu avec celles des variétés référencées afin d'obtenir une liste de propositions de cépages pouvant correspondre. La vérification est ensuite laissée au soin de l'ampélographe (TRUel et al., 1980).

L'utilisation optimale de ce système nécessite une bonne connaissance du fichier et des caractères descriptifs utilisés. C'est pourquoi une analyse de ces données a été envisagée. Les résultats obtenus permettent d'aborder avec une optique nouvelle les problèmes posés par l'ampélographie chez Vitis vinifera L.

\section{FICHIER ET SON UTILISATION}

\section{A. Description du fichier}

L'établissement de ce fichier a débuté en 1954. Il a été réalisé au domaine de Vassal (I.N.R.A.) et offre une image assez représentative de la collection de variétés de Vitis vinifera $\mathrm{L}$. qui existe sur ce domaine (TRUEL, 1985). Pour un même cépage plusieurs introductions ont pu être effectuées sans que l'on sache dans tous les cas s'il s'agissait ou non de clones différents. Aussi, chaque introduction est considérée $a$ priori comme un " exemplaire " distinct. En collection chaque « exemplaire » est représenté par 5 plants ( 5 souches) sur lesquels portent la description. Tous les « exemplaires » introduits sont, après identification transcrits dans ce fichier. Leur nombre varie selon les variétés de 1 à 93 et dépend des opportunités rencontrées lors de la collecte du matériel végétal (importance de l'aire de culture du cépage, de sa dispersion géographique et de sa variabilité clonale). La quantité d'exemplaires décrits par cépage ne peut donc pas être considérée comme la résultante d'un paramètre unique mais d'un ensemble de facteurs pouvant avoir des influences relatives variables.

Les descriptions ont été réalisées à partir de fiches d'observation et de feuilles d'herbier qui sont maintenant archivées. Elles constituent ainsi des éléments de référence auxquels on peut facilement se reporter.
Le schéma directeur qui a servi de base à ces descriptions est celui qui a été proposé par l'O.I.V. en 1951. En tout, 75 caractères ont été pris en compte ce qui représente 272 classes ou niveaux d'expression. Le nombre de classes varie en effet selon le caractère, de 2 à 9 mais se situe en moyenne entre 3 et 4 .

Les organes décrits sont principalement :

L'extrémité du rameau, les jeunes feuilles, la feuille adulte, les pétioles, le rameau, les vrilles, la fleur, la grappe et la baie. D'une façon générale, les caractères intéressent la forme, la couleur, la villosité, et l'importance du développement de ces différents organes (GALET, 1956-64, Descriptors for grape, 1983). On peut citer par exemple la découpure du limbe des feuilles $(1,3,5,7,9$ lobes et plus), la taille des grappes selon leur longueur (petite $<12 \mathrm{~cm}$, moyenne 12 $18 \mathrm{~cm}$ et grande $>18 \mathrm{~cm}$ ) ou encore la forme des baies (aplatie, arrondie, elliptique, ovoïde, troncovoïde, obovoïde, cylindrique ou arquée).

Lors de la description de l'échantillon de référence si une fluctuation entre 2 classes voisines d'un même caractère est décelée, celle-ci es prise en compte sans pondération. En effet, ce fichier a dans un premier temps été transcrit sur cartes perforées afin de pouvoir être traité par un procédé électromagnétique. L'appartenance à une classe était alors symbolisée par une perforation dans la colonne et au rang correspondant à cette classe. Cette façon d'opérer n'a pas permis d'estimer le pourcentage d'appartenance aux différentes classes.

Chaque description d'exemplaires est répertoriée par 6 chiffres : les 4 premiers représentent le cépage et les 2 derniers, l'exemplaire proprement dit.

Actuellement le fichier comporte 5226 exemplaires, soient 2076 variétés.

\section{B. Utilisation du fichier}

\section{Transcription sur support magnétique}

Du fait de l'importance de ce fichier et afin de pouvoir utiliser ces données sur le matériel informatique du centre (Mini 6), les cartes perforées ont été recopiées et sauvées sur une cartouche magnétique. Le code établi pour l'établissement de ces cartes, il y a 35 ans ne correspondant à aucun code logique actuel, celles-ci ont dû être lues en binaire. Un programme en langage Pascal réalisé par G. FRANCILLON (I.N.R.A., Biométrie, Montpellier) a alors permis de décoder ces données et de les rendre facilement utilisables.

Ainsi, tout exemplaire est maintenant symbolisé par un nombre de 6 chiffres et par 272 espaces pouvant prendre les valeurs 1 ou 0 selon l'appartenance ou non de l'exemplaire à la classe correspondante.

\section{Utilisation du fichier en vue de l'obtention d'une liste d'hypothèses}

La conception du programme permettant cette sélection a tenu compte principalement de deux contraintes :

- le nombre, l'ordre et le choix des classes de caractères sur lesquelles porte le questionnaire devaient pouvoir être variables et laissés à l'initiative de l'opérateur, 
- la sélection positive ou négative devait être possible afin de conserver ou non la fluctuation susceptible d'avoir été enregistrée lors de la description.

Un programme en langage Pascal tenant compte de ces 2 principes a ainsi été conçu (fig. 1). Pratiquement il permet une utilisation de la façon suivante :

Dans un premier temps l'utilisateur indique le nombre de classes sur lequel va porter son questionnaire. Puis il entre dans l'ordre de son choix les numéros de code des classes suivis de la valeur 1 ou 0 selon le mode de sélection envisagé (positif ou négatif).

Une fois ce travail effectué la présence de l'utilisateur n'est plus nécessaire. Les exemplaires sont lus et triés un par un, ce qui demande environ $35 \mathrm{mn}$. Cette lecture intéresse uniquement les classes choisies et s'effectue dans l'ordre envisagé.

Les exemplaires qui possèdent les niveaux d'expression retenus pour les caractères testés sont enregistrés dans des fichiers successifs dont les numéros correspondent aux numéros d'ordre des classes dans le questionnaire considéré. Dans le dernier fichier ne subsis- tent alors que les exemplaires ayant satisfait à tous les tris précédents. La liste d'exemplaires qui est contenue est alors considérée a priori comme la liste d'hypothèses pouvant correspondre à l'échantillon analysé.

Ce système consiste en fait à réaliser une succession d'intersections entre différents sous-ensembles d'exemplaires possédant les caractéristiques désirées.

Contrairement à une clé dichotomique rigide ce procédé offre une grande souplesse d'utilisation et permet une adaptation du questionnaire en fonction de l'échantillon considéré.

En conséquence la réussite d'une recherche dépend de trois facteurs :

- Il faut que la variété recherchée se trouve réellement dans le fichier.

- Il faut que l'échantillon sur lequel se base le questionnaire soit suffisamment représentatif.

- Il faut que le protocole du questionnaire soit judicieux.

Concernant la première condition, le nombre de cépages : 2076 , contenus dans ce fichier offre une

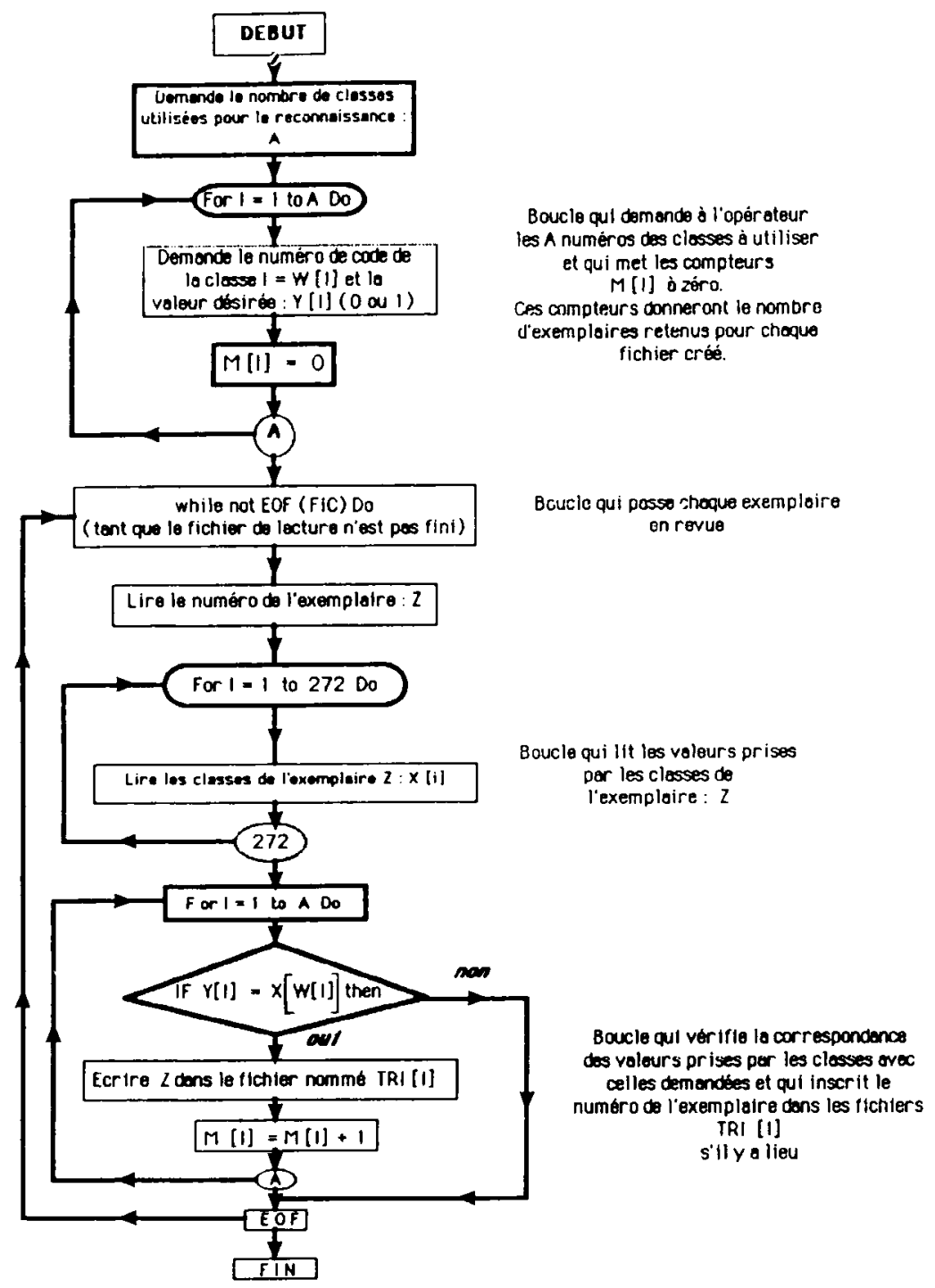


bonne garantie quant à la présence probable d'une variété dans le fichier de référence. Néanmoins il faut continuer à enrichir ce fichier.

La représentativité de l'échantillon est un facteur limitant extrêmement important auquel nous avons déjà fait allusion et qui doit être pris en considération.

Le dernier point est lié à l'utilisateur. De par la souplesse de ce système, c'est à lui que revient la responsabilité de l'établissement du questionnaire en fonction de la description de l'échantillon à tester. Généralement un questionnaire porte sur 10 à 15 classes de caractères et doit permettre d'obtenir ainsi une liste contenant 20 à 50 exemplaires.

$\mathrm{La}$ réussite de l'entreprise et l'obtention d'une bonne hypothèse résultent de la façon dont est conçu le questionnaire. Il faut pour cela avoir une bonne connaissance de la manière dont a été réalisé le fichier et être en mesure de pouvoir apprécier les principales propriétés des caractères ampélographiques utilisés : la sélectivité et la fiabilité (Truel, BoursiQuot, 1985).

En effet, il s'agit lors de ces tris d'éliminer le plus de cépages possibles ne correspondant pas à l'échantillon afin de limiter le nombre des hypothèses fausses tout en essayant de réduire au maximum le risque d'éliminer la bonne variété.

Un programme permettant de calculer, pour l'ensemble des exemplaires, la fréquence d'apparition de la valeur 1 dans les différentes classes de caractères a été élaboré afin de savoir comment ceux-ci avaient été pris en compte lors des descriptions et de pouvoir ainsi estimer leur sélectivité et leur fiabilité dans ce fichier. De plus, étant donné le nombre de cépages représentés et malgré le léger biais pouvant être engendré par la quantité variable d'exemplaires décrits par variété, les résultats obtenus fournissent une estimation de la répartition de la population de Vitis vinifera dans les classes des différents caractères ampélographiques, sans doute assez proche de celle que l'on obtiendrait si l'on était en mesure d'étudier cette population dans son ensemble.

- La sélectivité : un caractère n'est pas sélectif en soi mais une classe de caractère peut l'être.

Ainsi, parmi les résultats obtenus on peut citer par exemple :

classe $12-$ feuille adulte entière

$\mathrm{f}=0,05$

classe 13 - feuille adulte 3 lobes

$\mathrm{f}=0,28$

classe 14 - feuille adulte 5 lobes

$\mathrm{f}=0,83$

$f=0,35$

classe 15 - feuille adulte 7 lobes

$\mathrm{f}=0,13$

Pour ce caractère les classes 12 et 16 sont particulièrement sélectives : $5 \%$ seulement des exemplaires contenus dans ce fichier présentent la particularité d'avoir des feuilles entières et $13 \%$ ont des feuilles à 9 lobes ou plus.

Par ailleurs, la sélectivité d'une classe peut dépendre des tris précédents. En effet, il existe parfois des liaisons entre niveaux d'expression de caractères différents. On peut là encore illustrer cette remarque par un exemple. Le questionnaire suivant a été réalisé :

classe $12-$ feuille entière -0

classe 13 - feuille 3 lobes -0

classe 14 - feuille 5 lobes $-0:$ reste 593 exemplaires classe $20-$ sinus latéraux -1 : reste 584 exemplaires supérieurs très $\quad f=0,82$ profonds

On peut en conclure que le sous-ensemble formé par les exemplaires dont les feuilles ont 7 lobes et plus, est pratiquement inclus dans celui formé par les exemplaires à sinus latéraux supérieurs très profonds (fig. 2). En effet cette dernière classe a une fréquence de 0,82 et se rencontre donc chez $4285(0,82 \times 5226)$ exemplaires.

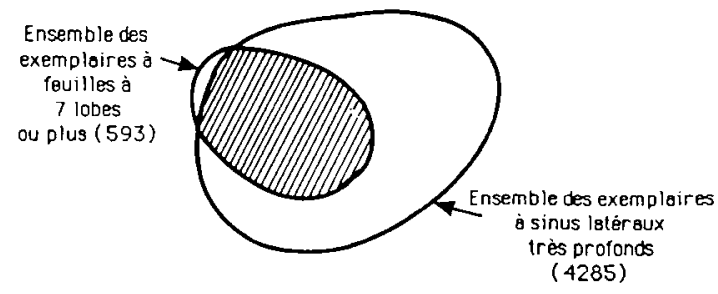

Figure 2

Schéma représentatif d'un tri qui utilise des modalités présentant un certain degré de liaison.

Diagram of a sort based on character values with a certain degree of relationship.

- La fiabilité : on considère qu'un caractère est fiable lorsque la fluctuation est faible. Le nombre de classes prises en compte lors de la description des exemplaires pour ces caractères est alors généralement égal à 1 . Il est possible d'estimer d'une façon globale cette caractéristique par la somme des fréquences des classes d'un caractère.

classe 87 - baie blanche

$$
\mathrm{f}=0,46
$$

classe 88 - baie grise

$\mathrm{f}=0,02$

classe 89 - baie rose

$\mathrm{f}=0,06$

classe 90 - baie rouge

$\mathrm{f}=0,04$

classe 91 - baie noire

$\mathrm{f}=0,43$

$\Sigma=1,01$

Le caractère couleur des baies est donc particulièrement fiable. Cependant, pour certains caractères comme par exemple le nombre de lobes de la feuille adulte, la fiabilité peut varier en fonction des cépages. On pourra alors apprécier cette caractéristique qu'en extrapolant a priori les observations réalisées sur l'échantillon à reconnaître.

A la vue de ces remarques on s'aperçoit que l'élaboration du questionnaire est une phase très délicate dans l'utilisation de ce système. L'utilisateur de cette méthode de tri devra donc avoir une bonne connaissance des problèmes ampélographiques et de la façon dont a été conçu ce fichier.

Ce système informatisé reproduit le procédé employé précédemment (trieuse de cartes) mais facilite énormément les manipulations, ce qui permet une utilisation bien meilleure de ces données.

\section{ANALYSE STATISTIQUE DES DONNÉES}

Etant donné l'importance et le grand nombre de variables utilisées une méthode d'analyse des données 
multidimensionnelles semblait toute indiquée. Sous ce terme on regroupe un ensemble de méthodes statistiques ayant pour but de fournir une représentation géométrique de l'information contenue dans le tableau analysé. L'approche statistique est dans ce cas purement descriptive. Elle permet de dégager les traits dominants de ce fichier et peut ainsi servir de base à des analyses plus fines.

\section{A. Traitement des données}

La méthode retenue a été l'analyse Factorielle des Correspondances (A.F.C.).

Cette analyse s'applique à des tableaux formés de variables qualitatives. Le fichier ampélographique est en effet un tableau de description logique formé de 0 et de 1 croisant 5226 individus avec 75 variables qualitatives (caractères) subdivisées en 272 modalités (classes ou niveaux d'expression). Afin de donner un poids identique aux variables et aux individus on a soumis à l'analyse factorielle des correspondances un tableau modifié dont la somme des valeurs associées aux modalités d'une variable est égale à 1 . Par exemple si un exemplaire présentait 2 niveaux d'expression pour un caractère descriptif, cette information notée initialement 2 fois par 1 a été transformée en $1 / 2$ et $1 / 2$.

Le nombre d'exemplaires décrits était également très variable selon les variétés. Aussi, pour ne pas favoriser les cépages représentés par un grand nombre d'exemplaires, un individu moyen a été créé à partir des exemplaires décrits pour chaque variété. C'est sur ce nouveau tableau logique contenant 2076 individus que l'A.F.C. a été réalisée (VOLLE, 1985).

Cette méthode descriptive est maintenant suffisamment connue (BENZECRI et al., 1973) pour que nous ne la présentions ici que succinctement. Elle permet d'obtenir la meilleure représentation visuelle (au sens d'un certain critère) et d'apprécier ainsi la proximité entre individus et variables (LEBART et al., 1982). Elle offre par des représentations planes la possibilité d'illustrer ces proximités et d'établir des relations entre des groupes d'individus et les caractères descriptifs de ces individus.

\section{B. Résultats et discussion}

Lors du dépouillement des résultats il convient d'étudier dans un premier temps les pourcentages d'inertie expliqués par les axes principaux. Ils donnent une idée de leur pouvoir descriptif respectif.

$$
\begin{aligned}
& \text { axe } 1=5,57 \% \\
& \text { axe } 2=4,19 \% \\
& \text { axe } 3=3,54 \% \\
& \text { axe } 4=2,42 \% .
\end{aligned}
$$

Ces pourcentages d'inertie peuvent paraître faibles, mais il faut remarquer que les 2 premiers axes apportent $9,8 \%$ de l'information, soit 13 fois plus que n'importe quel plan formé à partir des directions de 2 classes initiales (chacune renfermant 100/272= $0,37 \%$ d'information).
Etant donné le nombre très élevé d'individus et de classes de caractères il n'est malheureusement pas possible de reproduire ici intégralement les graphiques obtenus par l'A.F.C. Leur observation a permis tout d'abord d'étudier la répartition des différentes classes de caractères. Une signification des axes et de l'information qui est contenue peut ainsi être proposée. En effet, un caractère est d'autant plus corrélé avec un axe que ses classes s'allongent parallèlement à la direction de cet axe.

D'autre part, l'étude des graphiques faisant intervenir le troisième facteur n'a pas permis de déboucher sur une interprétation pratique et significative. Elle ne sera donc pas abordée ici.

Sur le graphique concernant les classes de caractère projetées dans le plan des axes 1 et 2 , on peut observer une répartition du nuage faisant intervenir 4 pôles principaux (fig. 3) et pouvant être définis ainsi :

- un pôle rassemblant les classes symbolisant une pigmentation anthocyanique prononcée pour différents organes et la présence d'une densité des poils dressés importante ;

- un pôle regroupant les classes représentant une densité des poils couchés forte pour les organes végétatifs :

- un pôle réunissant les classes correspondant à une absence de pigmentation anthocyanique et à une densité des poils dressés faible ou nulle ;

- et enfin un pôle rassemblant les classes représentant une densité des poils couchés faible ou nulle.
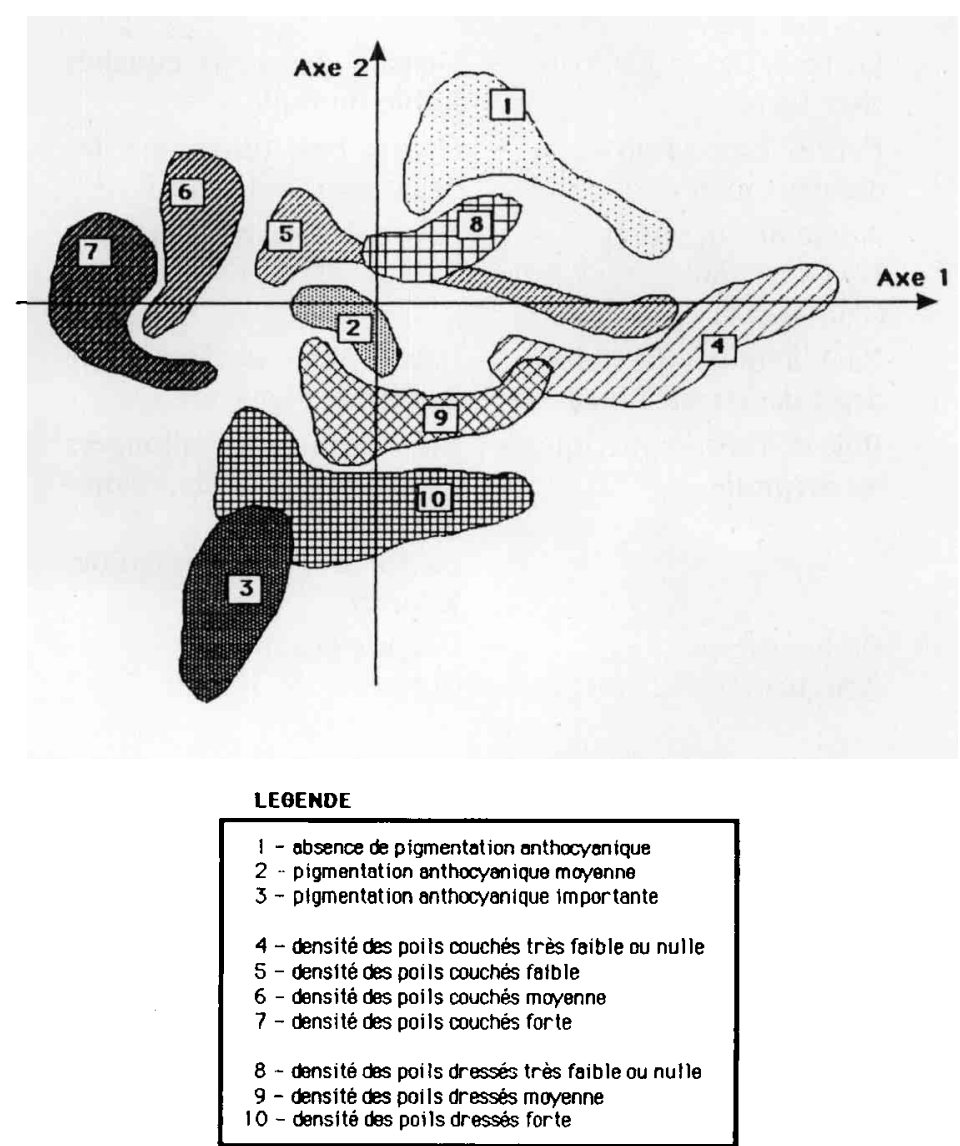

Figure 3

Représentation du nuage des classes de caractères.

Scatter diagram of the character values. 
Ces 4 sommets déterminent 2 directions qui correspondent pratiquement à celles des axes 1 et 2 .

On peut cependant noter une légère rotation vis-àvis de ces axes mais celle-ci est faible et les différents caractères s'allongent régulièrement le long des axes.

Ce sont donc des caractères relatifs à la densité des poils couchés qui ont surtout contribué à la formation de l'axe 1.

Quant à l'axe 2 ce sont des caractères liés à la couleur des organes et à la densité des poils dressés qui ont participé à son élaboration.

L'orthogonalité des deux axes fait que ces caractères peuvent être considérés comme indépendants. Ce sont eux qui dispersent le plus les individus. On aura donc tout intérêt à les employer en priorité lors de la construction d'une clé de détermination.

Ces observations sont confirmées par le tableau des contributions. On peut également en affinant cette étude faire ressortir d'autres caractères qui n'apportent qu'une contribution beaucoup plus faible, voire négligeable mais qui s'allongent cependant nettement le long de ces axes et qui peuvent donc présenter un certain degré de liaison. La mise en évidence de relations entre caractères différents peut alors faire entrevoir des groupes de cépages ayant en commun certaines caractéristiques et possédant ainsi un type de faciès particulier.

En se déplaçant dans le sens positif selon l'axe 1 on pourra observer une évolution respective des caractères suivants :

\section{AXE 1}

Densité des poils cou- - Densité des poils couchés chés forte faible ou nulle

Petite baie (moyenne $\rightarrow$ Grosse baie (moyenne des des deux axes $<14 \mathrm{~mm}$ ) deux axes $>18 \mathrm{~mm}$ )

Angle des nervures $\rightarrow$ Angle des nervures

$\mathrm{L}_{1}^{\prime} \mathrm{L}_{2}>180^{\circ} \quad \mathrm{LL}_{2}>90^{\circ}$

(voir fig. 4)

Baie à pulpe de faible $\rightarrow$ Baie à pulpe de degré élevé degré de fermeté (juteuse) de fermeté (charnue)

Baie de forme sphérique $\rightarrow$ Baie de formes allongées ou arrondie (elliptique, ovoïde, cylindrique)

ou particulières (obovoïde, arquée)

Petite grappe

(longueur $<12 \mathrm{~cm}$ )

- Grande grappe (longueur $>18 \mathrm{~cm}$ )

Ces caractères se rapportent essentiellement aux baies et aux grappes. Les organes végétatifs ne sont décrits que par leur villosité et l'angle des nervures de la feuille. Les zones définies par cet axe le seront principalement d'après leur type de production auquel viendra s'ajouter une légère caractéristique anatomique. Cet axe oppose donc deux populations bien distinctes au sein de Vitis vinifera : l'une contenant des cépages destinés à la cuve et généralement cotonneux et l'autre des variétés de table souvent glabres (GALET, 1967). Enfin il ne semble pas que le degré d'ouverture du sinus pétiolaire soit lié de façon directe à l'importance des angles formés par les nervures principales comme on aurait pu le penser a priori.

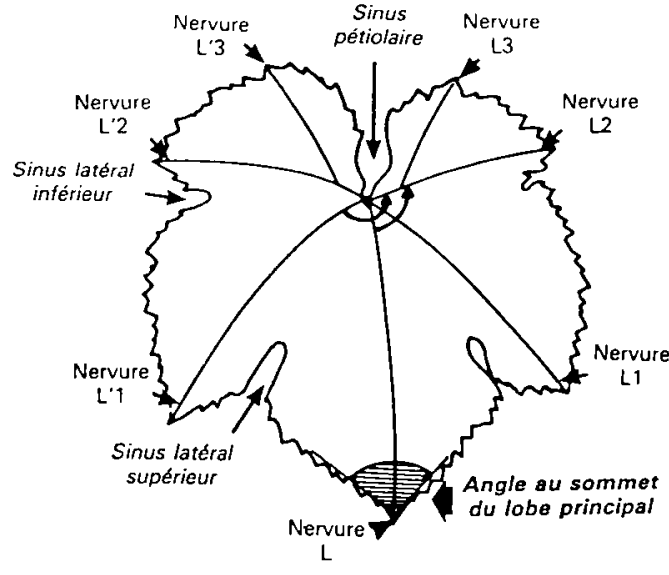

Figure 4

Schéma d'une feuille de vigne indiquant les caractères ampélographiques utilisés.

Diagram of a grapevine leaf showing the ampelographic characters used.

Au niveau de l'axe 2 on pourra suivre la progression suivante des caractères :

\section{AXE 2}

Pigmentation anthocya- $\rightarrow$ Absence de pigmentation nique prononcée (ner anthocyanique

vures, feuilles pétioles, rameaux)

Densité des poils dressés $\rightarrow$ Densité des poils dressés importante nulle ou faible

Sinus latéral supérieur $\rightarrow$ Sinus latéral supérieur peu profond (voir fig. 4) marqué

Sinus latéral inférieur $\rightarrow$ Sinus latéral inférieur peu profond (voir fig. 4) marqué

Feuille adulte découpée $\rightarrow$ Feuille adulte entière (nombre de lobes impor-

tant)

Petite feuille $\rightarrow$ Grande feuille

Angle au sommet $\mathrm{du} \rightarrow$ Angle au sommet du lobe lobe principal $<90^{\circ} \quad$ principal $>90^{\circ}$

Contrairement à l'axe 1 , ces caractères sont liés surtout à la description des feuilles. La relation qui est mise en exergue entre la découpure des feuilles et la profondeur des sinus latéraux est bine sûr évidente. Cependant celle qui semble exister entre la présence d'une pigmentation anthocyanique, la densité des poils dressés, la découpure de la feuille et sa dimension paraît plus originale et intéressante. On retrouve en effet vérifié au niveau de la population de Vitis vinifera étudiée ici, les remarques formulées par GRENAN \& TRUEL (1983) et NOZERAN et al. (1983) au sujet des caractères juvéniles.

L'axe 2 peut donc être défini comme l'axe du comportement juvénile des variétés.

En fonction de ces résultats il vaudra mieux lors de l'établissement d'un questionnaire utiliser en priorité les caractères décrivant et dispersant le mieux les variétés c'est-à-dire ceux qui apportent une contribution importante aux axes. Il faut cependant émettre quelques réserves vis-à-vis de certains caractères pou- 
vant présenter une fluctuation clonale importante (p.e. : la pigmentation anthocyanique des rameaux). Néanmoins, cette fluctuation a théoriquement été prise en compte dans le fichier de référence, ce qui devrait permettre malgré tout une utilisation de ces caractères. Il sera également préférable de choisir des caractères relativement indépendants entre eux (axes différents). Lors de l'utilisation de caractères liés au même axe, l'optimisation du choix se fera au regard de l'échantillon testé.

On peut maintenant envisager le problème de la dispersion des cépages en relation avec ce qui a été mis en évidence précédemment.

Sur le graphique concernant les axes 1 et 2 , le nuage de points représentant les individus forme un disque homogène. La densité de ce nuage est maximale dans la zone centrale et diminue régulièrement lorsqu'on s'éloigne du centre (fig. 5).

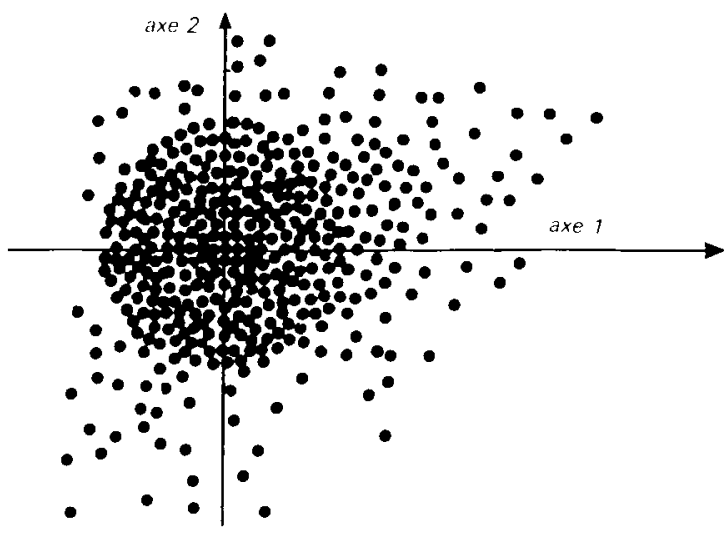

Figure 5

Représentation du nuage des individus.

Scatter diagram of the individuals.

Cette homogénéité et cette décroissance régulière de la densité montre qu'il n'existe pas de familles différenciées à l'intérieur de Vitis vinifera. Au contraire, entre types de cépages différents, on trouve un gradient dû au grand nombre de variétés et au formidable « brassage » qui a existé et qui continue de se réaliser au sein de cette espèce.

On peut cependant, en étudiant les proximités entre les individus et les caractères descriptifs et en combinant les interprétations des axes 1 et 2 , essayer de proposer 9 zones variétales. La figure 6 indique leurs positions respectives par rapport aux axes.

Les caractéristiques définissant ces zones et des exemples de cépages leur appartenant sont rassemblés dans le tableau 1. En plus des caractères principaux on peut trouver associés, d'une façon plus ou moins nette les caractères secondaires cités précédemment.

Les définitions des zones 4 et 2 sont extrêmement intéressantes à ce sujet. Ces 2 zones sont opposées sur le graphique. La zone 4 où se trouvent essentiellement des cépages teinturiers est définie par la présence d'une pigmentation anthocyanique prononcée sur les différents organes décrits et par une densité des poils dressés et couchés importante. Parmi les caractéristiques secondaires on peut citer : des petites baies, des petites grappes, des feuilles découpées et de faibles dimensions. Ce sont bien là des caractères liés à un

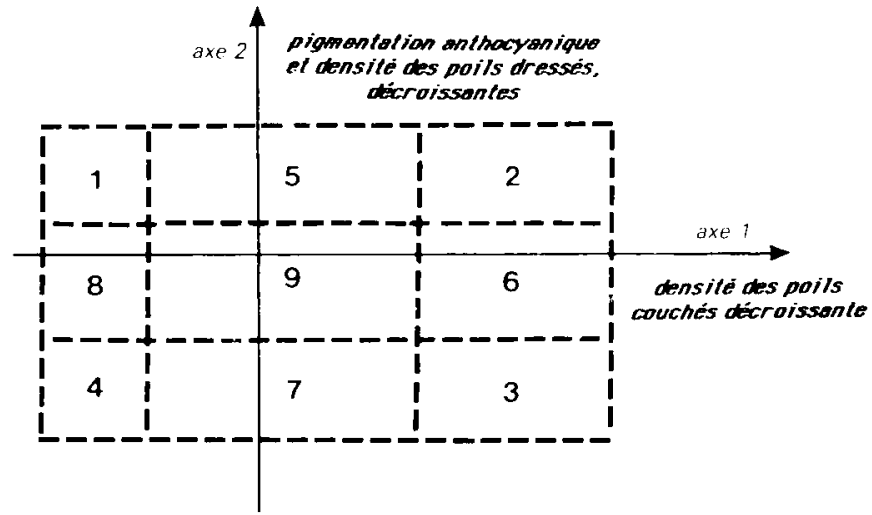

Figure 6

Schéma de proposition des zones variétales.

Diagram of proposed varietal zones.

faible degré d'évolution. Il faut préciser qu'un grand nombre «d'hybrides » Bouschet c'est-à-dire de cépages teinturiers, ont été obtenus par semis et descendent de la variété Teinturier du Cher. Ils sont réunis dans cette zone ce qui contribue à lui conférer ces caractéristiques.

\section{TABLEAU 1}

Zones variétales et exemples.

Varietal zones and examples.

\begin{tabular}{ccc}
\hline Zones & \multicolumn{1}{c}{ Caractéristiques } & \multicolumn{1}{c}{ Cépages } \\
\hline 1 & $\begin{array}{l}\text { Pigmentation anthocyanique } \\
\text { et densité des poils dressés } \\
\text { faibles, densité des poils cou- } \\
\text { chés importante }\end{array}$ & nier, Gros Maroc, Gerdeca \\
\hline
\end{tabular}

2 Pigmentation anthocyanique Crujillon, Gateta, Kouldet densité des poils dressés jinski, Luisa blanca, Obak faibles; densité des poils blanc, Opsimos Edessis couchés faible 49 Pirovano, Torralba

3 Pigmentation anthocyanique Aramon Bouschet $n^{\circ} 5$ et densité des poils dressés Assoued Zeme, Bruni n ${ }^{\circ} 100$, importantes ; densité des Gloria Hungariae, Heptapoils couchés faible kilo, Karmir Kakhani, 63 Pirovano

4 Pigmentation anthocyanique Carignan Bouschet, OEilet densité des poils dressés lade Bouschet $n^{\circ} 1$, Petit importantes; densité des poils Bouschet, Teinturier du couchés importante Cher

5 Pigmentation anthocyanique Humagne, Brachetto, Caret densité des poils dressés ricante, Ciliegiolo, Emerald faible Riesling, Grechetto, Grenache, Mauzac

6 Densité des poils couchés Cardinal, Datal, Dattier, faible Glacière, Molinera Gorda, Olivette, Ribol, Sultanine, Teta de Vacca

7 Pigmentation anthocyanique Beclan, Danlas, Gamay tein et densité des poils dressés turier, J. Bernath, Lacryma importante Christi, Lival, San Antoni

8 Densité des poils couchés Aubun, Balzac blanc, Caliimportante tor, Mourvèdre, OEillade, Pinot Mèunier, Plavai, Tibouren

9 Caractéristiques intermédiaires 
Dans la zone 2 au contraire on trouve des cépages ayant subi une pression de sélection plus grande, des cépages plus "spécialisés " avec en particulier des variétés blanches de table. Les organes végétatifs sont verts et glabres. Les feuilles sont moins découpées, plus grandes, avec un angle des nervures $\mathrm{LL}_{2}$ inférieur à $90^{\circ}$, les baies et les grappes sont plus grosses.

La représentation graphique obtenue grâce à cette analyse statistique offre donc une image de l'évolution variétale qui existe au sein de Vitis vinifera.

La densité est par ailleurs maximale dans les zones 8,5 (et 9), ce qui est à mettre en relation avec la dominance et la stabilité des caractères qui les définissent.

L'importance de la zone intermédiaire 9, qui ne possède pas de caractéristiques propres est significative de la difficulté qui existe à établir des groupes de cépages à l'intérieur de Vitis vinifera... Une analyse plus poussée permettra peut être de réduire cette aire sans définition précise.

\section{CONCLUSION}

Un tel exemple montre bien l'aide que peut apporter l'informatique dans l'étude et la gestion d'un fichier de cette importance.
Le traitement par l'ordinateur permet maintenant une utilisation réactualisée et grandement facilitée de cette banque de données ampélographiques. C'est dorénavant un outil qui peut être une aide non négligeable pour la reconnaissance des variétés dans la mesure où il sera employé à bon escient. En particulier cela permettra d'avoir des indications précieuses pour déterminer si une nouvelle variété introduite en collection ne s'y trouve pas déjà sous un autre nom.

L'analyse statistique réalisée sur ce fichier a fourni des informations sur le rôle et l'intérêt des différents caractères ampélographiques utilisés. Une orientation dans leur choix a ainsi été rendue possible. La mise en évidence d'association entre caractères différents a permis également de proposer des regroupements de cépages possédant en commun certaines particularités.

L'intérêt de ces regroupements réside dans le fait qu'ils offrent une image de l'évolution qui peut exister au sein de Vitis vinifera. De plus leurs caractéristiques sont non seulement liées à des critères morphologiques mais aussi à des types de production ce qui les rapproche par certains aspects aux groupes géographiques et écologiques (proles) définis par NEGRUL en 1938 (in LEVADOUX, 1956.

Recu le 8 janvier 1986. Accepté le 14 septembre 1986.

\section{RÉFÉRENCES BIBLIOGRAPHIQUES}

Anonyme, 1983. Descriptors for grape. International Board for Plant Genetic Resources, Rome, 93 p.

Benzecri J. P. et collaborateurs, 1973. L'analyse des données $t, 2$. $L$ 'analyse des correspondances. Ed. Dunod, Paris.

Branas J., 1959. Méthodes de description ampélographique et leur valeur pour l'identification des variétés. Bul. O.I.V., 1959, 31 (335), 18-41.

Galet P., 1956-1964. Cépages et Vignobles de France $(4$ t.). Imprimerie Dehan, Montpellier, 3500 p.

Galet P., 1967. Thèse. Recherches sur les méthodes d'identification et de classification des Vitacées des zones tempérées. 526 p., Montpellier.

Grenan S., Truel P., 1983. Observations sur un aspect de la variabilité constatée au cours de la multiplication végétative de variétés de vigne issues de semis de Vitis vinifera L. Agronomie, 1983, 3 (7), 675-680.

Lebart L., Morineau A., Fenelon J. P., 1982. Traitement des données statistiques, 305-328, Dunod, Paris, $2^{\mathrm{e}}$ édition.
Levadoux L., 1956. Les populations sauvages et cultivées de Vitis vinifera L. Annales de l'Amélioration des Plantes, 1956, 1, 59-118.

Nozeran R., Grenan S., Truel P., 1983. Morphogenèse à partir du stade juvénile de Vitis vinifera $\mathrm{L}$. issu de graine ou de culture in vitro. Agronomie, 1983 (3), 681-684.

Truel P., 1985. Catalogue des variétés de vigne en collection à la Station de Recherches Viticoles de Montpellier. I.N.R.A., 1985, $129 \mathrm{p}$.

Truel P., Boursiquot J. M., 1985. Etudes sur les matériel introduit dans les collections ampélographiques en vue de son identification et de la recherche des synonymes. $4^{e}$ Symposium International de Génétique de la vigne, 1985. Italie.

Truel P., Rennes C., Domergue P., 1980. Identification dans les collections de vignes. $3^{e}$ Symposium International de Génétique de la vigne, 1980, Etats-Unis.

Volle M., 1985. Analyse des données. Ed. ECONOMICA, p. 130205. Collection E.S.A., $3^{\mathrm{e}}$ édition. 